\title{
Synthesis of novel unsymmetrically substituted 1,4-dihydropyridines and separation of the enantiomers of racemic 1,4-dihydropyridine containing isothioureido group
}

\author{
Moshtaghi Z. A.*, Raisossadat O. M., and Sadeghi Sh. Q. \\ Chemistry Department, Faculty of Science; Azarbaijan University of Tarbiat Moallem, \\ Tabriz Iran \\ E-mail:adelehmz@yahoo.com
}

\begin{abstract}
Unsymmetrical 1,4-dihydropyridine esters 3a and 3d-h were synthesized from the symmetrical precursors 1a-c through the intermediacy of 2-bromomethyl derivatives 2a-c. Chiral acid o,ódibenzoyl-L-tartaric acid was used to separate the enantiomers of racemic 1,4-dihydropyridine 3a.
\end{abstract}

Keywords: 1,4-Dihydropyridines, calcium channel blockers, enantiomer separation

\section{Introduction}

The 1,4-dihydropyridine $\mathrm{Ca}^{2+}$ channel blockers are clinically significant antihypertensive drugs ${ }^{1-3}$ and have been immensely valuable as molecular tools with which probe structural and functional aspects of $\mathrm{Ca}^{2+}$ channel function. ${ }^{4,5}$

Most of the 1,4-dihydropyridines were prepared via the Hantzsch procedure. ${ }^{6}$ This procedure is simple, and isolation of the product is generally straightforward but it works moderately well for symmetrical dihydropyridines, and the yield of the desired products decreases very rapidly for asymmetrically substituted dihydropyridines. ${ }^{7}$ Symmetrically substituted dihydropyridines such as nifedipine are achiral compounds. Different substituents in compounds of the second-generation such as amlodipine, nitrendipine and nicardipine lead to chiral derivatives whose enantiomers differ in their pharmacological effects. In the case of the calcium antagonists, the differences are quantitative. However, the introduction of certain substituens in position 3 or 5 can lead to calcium agonists. The enantiomers exhibit opposite activity, one of them acting as agonist, the other as an antagonist. In this work we synthesized some new derivatives of unsymmetrically substituted 1,4-dihydropyridine rings. The compounds 3a and 3d-h were synthesized by using nucleophilic attack of thiourea, 2-mercapto-4,6-dimethyl 
pyrimidine, 3,5-dimethylPyrazol, ammonium thiocyanate and sodium azide on the 2bromomethyl-1,4-dihydropyridines $\mathbf{2 a - c .}$

In view of chirality to pharmacological activity, the present article will describe the separation of the enantiomers of S-[(6-methyl-3,5-dicarboethoxy-4-(3-nitrophenyl) 1,4dihydropyridin-2-yl)-methyl]-isothiourea.

\section{Results and Discussion}

Synthesis was started by Hantzsch reaction of ethyl acetoacetate with appropriate aldehyde and ammonia in refluxing ethanol, which afforded the 1,4-dihydropyridines 1a-c. Reaction of 1,4dihydropyridines 1a-c with 1.1 equivalents of pyridinium bromide perbromide in dichloromethane/pyridine at $-20{ }^{\circ} \mathrm{C}$ for 45 minutes afforded the crude products $\mathbf{2 a - c}$ as a yellow gum. We have published before the synthesis of 2a [2-bromomethyl-3,5-dicarboethoxy-6methyl-4-(3-nitrophenyl)-1,4-dihydropyridine] in high yield $^{8}$ by modifying the literature methods. ${ }^{7,9,10}$ Without further purification these brominated adducts were coupled with a range of nucleophiles at different conditions to give 2-substituted 1,4-dihydropyridines 3a and 3d-h (see table 1).

In the reaction of $\mathbf{2 a}$ with thiourea in refluxing ethanol for $5 \mathrm{~h}$, evaporation of solvent and recrystallization from EtOAc/Hex, isothiouronium salt $\mathbf{3 a}$ is formed. Transformation of isothiouronium salt $\mathbf{3 a}$ into its isothiourea $\mathbf{3 b}$, as free base, carried out by treatment of $\mathbf{3 a}$ in $\mathrm{CH}_{2} \mathrm{Cl}_{2} / \mathrm{H}_{2} \mathrm{O}$ with $\mathrm{Na}_{2} \mathrm{CO}_{3}$ with vigorous stirring, at room temperature. ${ }^{11}$ The $\mathrm{C}-4$ carbon atom of 1,4-dihydropyridines is a prochiral atom. When at least one of the sunstituents, bound to the C-2 and C-3 carbon atoms, is different from those on the symmetric C-6 and C-5 positions of ring, the $\mathrm{C}-4$ carbon atom is chiral and the compounds are racemates. Meanwhile compound $\mathbf{3 b}$ with different substituents at C-2 and C-6 is a racemic mixture. In order to prepare the diasteromeric salts of $\mathbf{3 b}$, the mixture of $\mathbf{3 b}$ and (1S)-(+)-camphor-10-sulfonic acid in $\mathrm{CH}_{3} \mathrm{CN}$ was refluxed for $4 \mathrm{~h}$, but the product obtained was the thiol derivative 3c. However, Optical resolution of $( \pm)-3 \mathbf{b}$ by salification with $o$,ó-dibenzoyl-L-tartaric acid and recrystallization of diastereomeric salts, then hydrolysis of crystallized diastereomer (-)-4 in $\mathrm{CH}_{2} \mathrm{Cl}_{2} / \mathrm{H}_{2} \mathrm{O}$ with $\mathrm{NaHCO}_{3}$ gave (-)-3b with $65 \%$ yield. On the other hand, reaction of isothiouronium salt (-)-4 with allyl bromide in the presence of base produces S-alkylated derivative (-)-3i (Scheme 1). 
Table 1. Compounds 3<smiles>[R]CC1=C(C(=O)OC)C(c2cccc([R])c2)C(C(=O)OCc2ccccc2)=C(C)N1</smiles>

\begin{tabular}{|c|c|c|c|c|}
\hline Entry & Product & $\mathrm{R}^{1}$ & $\mathrm{R}^{2}$ & Yield $(\%)$ \\
\hline 1 & $3 a$ & $-\mathrm{SC} \ \mathrm{NH}_{2}^{+} \mathrm{Br}^{-}$ & $3-\mathrm{NO}_{2}$ & 80 \\
\hline 2 & $3 b$ & $-\mathrm{SC} \underset{\mathrm{NH}}{-\mathrm{NH}_{2}}$ & $3-\mathrm{NO}_{2}$ & 60 \\
\hline 3 & $3 \mathrm{c}$ & - SH & $3-\mathrm{NO}_{2}$ & 45 \\
\hline 4 & $3 d$ & & $2-\mathrm{OCH}_{3}$ & 57 \\
\hline 5 & $3 e$ & $-\mathrm{N}_{3}$ & $2-\mathrm{Cl}$ & 35 \\
\hline 6 & $3 f$ & $-\mathrm{SCN}$ & $2-\mathrm{Cl}$ & 45 \\
\hline 7 & $3 g$ & $\mathrm{H}_{3} \mathrm{C}$ & $2-\mathrm{Cl}$ & 49 \\
\hline 8 & $3 h$ & $\mathrm{H}_{3} \mathrm{C}$ & $3-\mathrm{NO}_{2}$ & 35 \\
\hline 9 & $3 \mathrm{i}$ & $-\mathrm{S} \mathrm{CH}_{2} \mathrm{CH}=\mathrm{CH}_{2}$ & $3-\mathrm{NO}_{2}$ & 45 \\
\hline
\end{tabular}




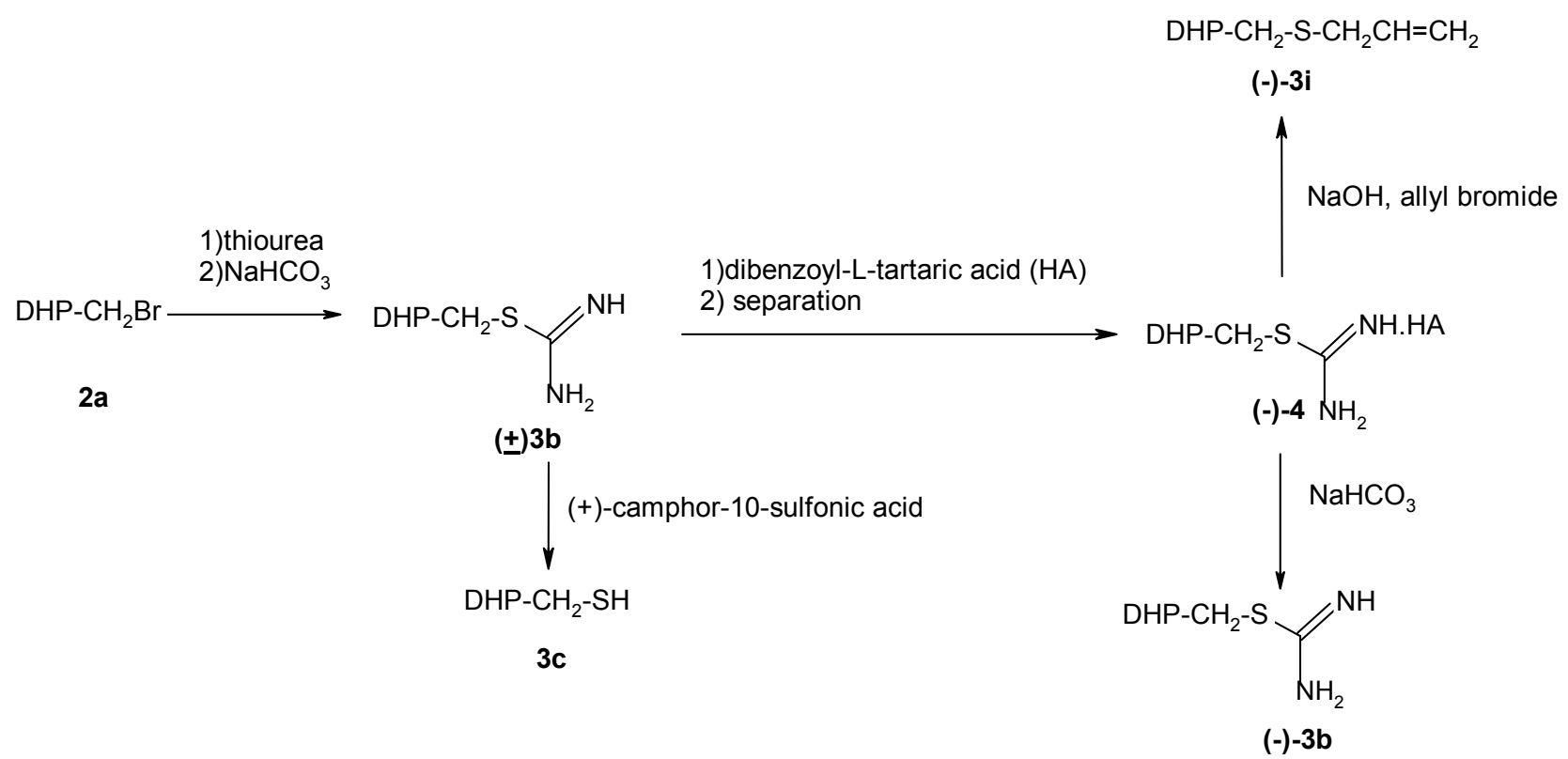

Scheme 1

\section{Experimental Section}

2-[(4,6-Dimethylpyrimidin-2-yl)thio-]-methyl-3,5-dicarboethoxy-6-methyl-4-(2-methoxyphenyl)-1,4-dihydropyridine (3d). A mixture of 2-bromomethyl-3,5-dicarboethoxy-6-methyl-4(2-methoxyphenyl)-1,4-dihydropyridine 2c (obtained from $1.39 \mathrm{mmol}$ of 1c), 2-mercapto-4,6dimethyl pyrimidine $(0.21 \mathrm{~g}, 1.53 \mathrm{mmol})$ and ethanol $(20 \mathrm{ml})$ was heated to reflux for $2 \mathrm{~h}$ and then evaporated. The residue was partitioned between $\mathrm{CH}_{2} \mathrm{Cl}_{2}(30 \mathrm{ml})$ and saturated $\mathrm{Na}_{2} \mathrm{CO}_{3}$ solution, and the organic layer washed with water, dried over $\mathrm{Na}_{2} \mathrm{SO}_{4}$ and evaporated. Recrystallization of crude product from 2-propanol furnished 3d $(0.38 \mathrm{~g}, 57 \%)$ as yellow crystals. M.p. $142{ }^{\circ} \mathrm{C}$, IR (KBr) $\bar{v}=3423$ (br. m), 3060 (w), 2977-2927 (m), 1686(s), 1643 (m), 1585 (s), 1489 (s), 1310 (s), 1288 (m), 1103(s), 747 (w) cm ${ }^{-1} ;{ }^{1} \mathrm{H}$ NMR (400 MHz, CDCl $): \delta=$ 1.17 (t, $J=6.47 \mathrm{~Hz}, 3 \mathrm{H}, \mathrm{CH}_{3}$ ester), 1.20 (t, $J=6.12 \mathrm{~Hz}, 3 \mathrm{H}, \mathrm{CH}_{3}$ ester), 2.21 (s, 3H, $\left.\mathrm{CH}_{3}-6\right), 2.47$ (s, 6H, $2 \times \mathrm{CH}_{3}$ pyrimidine), 3.72(s, 3H, O- $\left.\mathrm{CH}_{3}\right), 3.99-4.09\left(\mathrm{~m}, 4 \mathrm{H}, 2 \times \mathrm{CH}_{2}\right.$ ester), $4.49(\mathrm{AB}$ quartet, $\left.J=14.34 \mathrm{~Hz}, 2 \mathrm{H}, \mathrm{CH}_{2}-2\right), 5.27$ (s, 1H, C(4)-H), 6.76-7.82 (m, 3H, Ar-H, H-5 pyrimidine), 7.09 (dt, $\left.J_{l}=7.74 \mathrm{~Hz}, J_{2}=1.46 \mathrm{~Hz}, 1 \mathrm{H}, \mathrm{Ar}-\mathrm{H}\right), 7.24\left(\mathrm{dd}, J_{l}=7.91 \mathrm{~Hz}, J_{2}=1.64 \mathrm{~Hz}, 1 \mathrm{H}\right.$, Ar-H), 8.55 (s, 1H, NH) ppm; anal. calcd for $\mathrm{C}_{26} \mathrm{H}_{31} \mathrm{~N}_{3} \mathrm{O}_{5}: \mathrm{C}, 62.75 ; \mathrm{H}, 6.27 ; \mathrm{N}, 8.44$. Found: $\mathrm{C}$, $62.92 ; \mathrm{H}, 6.05 ; \mathrm{N}, 8.36$.

2-Azidomethyl -3,5-dicarboethoxy-6-methyl-4-(2-chlorophenyl)-1,4-dihydropyridine (3e). A mixture of 2-bromomethyl-3,5-dicarboethoxy-6-methyl-4-(2-chlorophenyl)-1,4-dihydropyridine $\mathbf{2 b}$ (obtained from $1.37 \mathrm{mmol}$ of $\mathbf{1 b})$, sodium azide $(0.10 \mathrm{~g}, 1.15 \mathrm{mmol})$ and ethanol $(20 \mathrm{ml})$ was heated to reflux for $24 \mathrm{~h}$ and then evaporated. The residue was partitioned between $\mathrm{CH}_{2} \mathrm{Cl}_{2}$ $(20 \mathrm{ml})$ and $2 \mathrm{M} \mathrm{HCl}$ solution, and the organic layer washed with water, dried over $\mathrm{Na}_{2} \mathrm{SO}_{4}$ and 
evaporated. Recrystallization of crude product from EtOH furnished 3 e $(0.22 \mathrm{~g}, 35 \%)$ as yellow crystals.

IR $(\mathrm{KBr}) \bar{v}=3312$ (s), 3078 (w), 2976-2901 (m), 2115 (m), 1699 (s), 1509 (s), 1249 (s), 1204 (s), 1095 (s), 753 (s) cm. ${ }^{-1}{ }^{1} \mathrm{H}$ NMR (400 MHz, $\mathrm{CDCl}_{3}$ ): $\delta=1.06$ (t, $J=7.12 \mathrm{~Hz}, 3 \mathrm{H}, \mathrm{CH}_{3}$ ester), 1.24 (t, J=7.02 Hz, 3H, $\mathrm{CH}_{3}$ ester), 2.32 (s, 3H, $\left.\mathrm{CH}_{3}-6\right), 3.71$ (q, J=7.02 Hz, 2H, $\mathrm{CH}_{2}$ ester), 3.97 (q, J=7.10 Hz, 2H, $\mathrm{CH}_{2}$ ester), 4.53 (s, 2H, $\left.\mathrm{CH}_{2}-2\right), 5.37$ (s, $\left.1 \mathrm{H}, \mathrm{C}(4)-\mathrm{H}\right), 7.08$ (dt, $J_{l}=7.59 \mathrm{~Hz}$, $\left.J_{2}=1.68 \mathrm{~Hz}, 1 \mathrm{H}, \mathrm{Ar}-\mathrm{H}\right), 7.18\left(\mathrm{dt}, J_{I}=7.48 \mathrm{~Hz}, J_{2}=1.12 \mathrm{~Hz}, 1 \mathrm{H}, \mathrm{Ar}-\mathrm{H}\right), 7.26\left(\mathrm{dd}, J_{I}=2.89 \mathrm{~Hz}\right.$, $\left.J_{2}=1.27 \mathrm{~Hz}, 1 \mathrm{H}, \mathrm{Ar}-\mathrm{H}\right), 7.28\left(\mathrm{dd}, J_{l}=3.24 \mathrm{~Hz}, J_{2}=1.43 \mathrm{~Hz}, 1 \mathrm{H}, \mathrm{Ar}-\mathrm{H}\right), 8.12(\mathrm{~s}, 1 \mathrm{H}, \mathrm{NH}) \mathrm{ppm}$.

\section{2-Thiocyanatomethyl-3,5-dicarboethoxy-6-methyl-4-(2-chlorophenyl)-1,4-dihydropyridine}

(3f). A mixture of 2-bromomethyl-3,5-dicarboethoxy-6-methyl-4-(2-chlorophenyl)-1,4dihydropyridine $\mathbf{2 b}$ (obtained from $1.37 \mathrm{mmol}$ of $\mathbf{1 b}$ ), ammonium thiocyanate $(0.12 \mathrm{~g}, 1.51$ mmol) and ethanol $(20 \mathrm{ml})$ was heated to reflux for $3 \mathrm{~h}$ and then evaporated. The residue was partitioned between $\mathrm{CH}_{2} \mathrm{Cl}_{2}(20 \mathrm{ml})$ and $2 \mathrm{M} \mathrm{HCl}$ solution, and the organic layer washed with water, dried over $\mathrm{Na}_{2} \mathrm{SO}_{4}$ and evaporated. Recrystallization of crude product from dibuthyl ether furnished 3f (0.33 g, 45\%) as yellow crystals. M.p. $157{ }^{\circ} \mathrm{C}, \mathrm{IR}(\mathrm{KBr}) \overline{\mathrm{v}}=3351$ (s), 3057 (w), 2979-2937 (m), 2156 (s), $1686(\mathrm{~s}), 1651$ (m), 1625 (m), 1504 (s), 1281 (s), 1208 (s), 1095 (s), 1048 (s), 757(s) $\mathrm{cm}^{-1} ;{ }^{1} \mathrm{H}$ NMR (400 MHz, $\mathrm{CDCl}_{3}$ ): $\delta=1.22$ (t, $J=7.01 \mathrm{~Hz}, 6 \mathrm{H}, 2 \times \mathrm{CH}_{3}$ ester), $2.36\left(\mathrm{~s}, 3 \mathrm{H}, \mathrm{CH}_{3}-6\right)$, 4.05-4.14(m, 6H, $2 \times \mathrm{CH}_{2}$ ester and $\left.\mathrm{CH}_{2}-2\right), 5.43(\mathrm{~s}, 1 \mathrm{H}, \mathrm{C}(4)-\mathrm{H}), 6.75(\mathrm{~s}$, $1 \mathrm{H}, \mathrm{NH}), 7.09\left(\mathrm{dt}, J_{l}=7.58 \mathrm{~Hz}, J_{2}=1.52 \mathrm{~Hz}, 1 \mathrm{H}, \mathrm{Ar}-\mathrm{H}\right), 7.17\left(\mathrm{dt}, J_{l}=7.48 \mathrm{~Hz}, J_{2}=1.09 \mathrm{~Hz}, 1 \mathrm{H}, \mathrm{Ar}-\right.$ H), 7.26(dd, $\left.J_{I}=7.57 \mathrm{~Hz}, J_{2}=1.11 \mathrm{~Hz}, 1 \mathrm{H}, \mathrm{Ar}-\mathrm{H}\right), 7.40\left(\mathrm{dd}, J_{l}=6.23 \mathrm{~Hz}, J_{2}=1.52 \mathrm{~Hz}, 1 \mathrm{H}, \operatorname{Ar}-\mathrm{H}\right)$ ppm; Anal. Calcd for $\mathrm{C}_{21} \mathrm{H}_{24} \mathrm{~N}_{2} \mathrm{O}_{5} \mathrm{~S}: \mathrm{C}, 57.01 ; \mathrm{H}, 5.02 ; \mathrm{N}, 6.65$. Found: C, 57.34; H, 5.41; N, 6.50 .

2-(3,5-dimethyl Pyrazol-1-yl)-methyl-3,5-dicarboethoxy-6-methyl-4-(2-chlorophenyl)-1,4dihydropyridine (3g). A solution of 2-bromomethyl-3,5-dicarboethoxy-6-methyl-4-(2chlorophenyl)-1,4-dihydropyridine $\mathbf{2 b}$ (obtained from $1.54 \mathrm{mmol}$ of $\mathbf{1 b}$ ) in THF (15 ml) was added via a cannula, to a magnetically stirred solution of $\mathrm{NaPz}$ in $\mathrm{THF}$ at room temperature. $\mathrm{NaPz}$ was prepared in situ from $\mathrm{PzH}$ (3,5-dimethyl pyrazol) $(0.15 \mathrm{~g}, 1.51 \mathrm{mmol})$ and $\mathrm{NaH} 80 \%$ $(0.048 \mathrm{~g}, 1.60 \mathrm{mmol})$ in THF $(15 \mathrm{ml})$ at room temperature under argon atmosphere. The mixture was stirred at room temperature for $3 \mathrm{~h}$ and then evaporated. The residue was partitioned between $\mathrm{CH}_{2} \mathrm{Cl}_{2}$ and $\mathrm{HCl} 2 \mathrm{M}$, the organic layer washed with saturated $\mathrm{Na}_{2} \mathrm{CO}_{3}$ solution and water, dried over $\mathrm{Na}_{2} \mathrm{SO}_{4}$ and evaporated. Recrystallization of crude product from ethanol gives $\mathbf{3 g}(0.31 \mathrm{~g}$, 49\%) as milky crystals. M.p. 103-104 ${ }^{\circ} \mathrm{C}$, IR (KBr) $\bar{v}=3270$ (s), 3095 (w), 2980-2950 (m), 1692 (s), 1652 (m), 1620 (m), 1551 (s), 1510 (s) ,1280 (s), 1203(s), 1097(s), 752 (s) cm ${ }^{-1} ;{ }^{1} \mathrm{H}$ NMR $\left(400 \mathrm{MHz}, \mathrm{CDCl}_{3}\right): \delta=1.21\left(\mathrm{t}, J=7.09 \mathrm{~Hz}, 3 \mathrm{H}, \mathrm{CH}_{3}\right.$ ester), 1.25 (t, $J=7.13 \mathrm{~Hz}, 3 \mathrm{H}, \mathrm{CH}_{3}$ ester), $2.20\left(\mathrm{~s}, 3 \mathrm{H}, \mathrm{CH}_{3}\right.$ pyrazol), 2.22(s, 3H, $\mathrm{CH}_{3}$ pyrazol), $2.29\left(\mathrm{~s}, 3 \mathrm{H}, \mathrm{CH}_{3}-6\right), 4.06-4.20(\mathrm{~m}, 4 \mathrm{H}$, $2 \times \mathrm{CH}_{2}$ ester), $5.38\left(\mathrm{~d}, J=16 \mathrm{~Hz}, 1 \mathrm{H}, \mathrm{CH}_{2}-2\right), 5.46(\mathrm{~s}, 1 \mathrm{H}, \mathrm{C}(4)-\mathrm{H}), 5.72\left(\mathrm{~d}, J=16 \mathrm{~Hz}, 1 \mathrm{H}, \mathrm{CH}_{2}-2\right)$, 5.91(s, $1 \mathrm{H}, \mathrm{NH}), 7.05-7.37\left(\mathrm{~m}, 5 \mathrm{H}, \mathrm{Ar}-\mathrm{H}\right.$, Pyrazol-H) ppm; Anal. Calcd for $\mathrm{C}_{25} \mathrm{H}_{31} \mathrm{~N}_{3} \mathrm{O}_{5}$ : C, 62.94; H, 6.16; N, 9.17. Found: C, 62.92; H, 6.51; N, 9.19.

2-(3,5-dimethyl Pyrazol-1-yl)-methyl-3,5-dicarboethoxy-6-methyl-4-(3-nitrophenyl)-1,4dihydropyridine $\mathbf{3 h}$. The compound (3h) was synthesized with 2-bromomethyl-3,5- 
dicarboethoxy-6-methyl-4-(3-nitrophenyl)-1,4-dihydropyridine 2a as above method. M.p. 157$158^{\circ} \mathrm{C}$, IR (KBr) $\bar{v}=3293(\mathrm{~m}), 3100(\mathrm{w}), 2980-2850$ (m), 1687 (s), 1652 (m), 1620 (m), 1528 (s), 1499 (s) ,1350 (s), 1273 (s), 1203(s), $1102(\mathrm{~s}), 715$ (s) $\mathrm{cm}^{-1} ;{ }^{1} \mathrm{H}$ NMR $\left(400 \mathrm{MHz}, \mathrm{CDCl}_{3}\right): \delta=$ $1.23\left(\mathrm{~m}, 6 \mathrm{H}, 2 \times \mathrm{CH}_{3}\right.$ ester), 2.22-2.62(m, $9 \mathrm{H}, 2 \times \mathrm{CH}_{3}$ pyrazol and $\left.\mathrm{CH}_{3}-6\right), 4.06-4.20(\mathrm{~m}, 4 \mathrm{H}$, $2 \times \mathrm{CH}_{2}$ ester), $5.12(\mathrm{~s}, 1 \mathrm{H}, \mathrm{C}(4)-\mathrm{H}), 5.56\left(\mathrm{AB}\right.$ quartet, $\left.J=16 \mathrm{~Hz}, 2 \mathrm{H}, \mathrm{CH}_{2}-2\right), 5.91(\mathrm{~s}, 1 \mathrm{H}, \mathrm{NH})$, 7.37(m, 1H, Ar-H), 7.37(m, 1H, Ar-H), 7.49(m, 1H, Ar-H), 7.61(m, 1H, Ar-H), 8.08(m, 1H, ArH) ppm; Anal. Calcd for $\mathrm{C}_{24} \mathrm{H}_{28} \mathrm{~N}_{4} \mathrm{O}_{6}$ : C, 61.53; H, 5.98; N, 11.96. Found: C, 61.73; H, 6.07; N, 12.01 .

$S$-[(6-Methyl-3,5-dicarboethoxy-4-(3-nitrophenyl)1,4-dihydropyridin-2-yl)-methyl]isothiouronium o,ó-dibenzoyl-L-tartarate (-)-4. A mixture of ( \pm )-S-[(6-methyl)-3,5dicarboethoxy-4-(3-nitrophenyl)1,4-dihydropyridin-2-yl)-methyl]-isothiourea $3 \mathbf{b}(0.12 \mathrm{~g}, \quad 0.26$ $\mathrm{mmol}), o$,ó-dibenzoyl-L-tartaric acid $(0.04 \mathrm{~g}, 0.13 \mathrm{mmol})$ and isopropanol is heated to the reflux temperature for $2.5 \mathrm{~h}$. After cooling at room temperature, the solution is cooled to $0{ }^{\circ} \mathrm{C}$ for $24 \mathrm{~h}$. Recrystallization of the obtained crystalls from EtOAc/Hex furnished (-)-S-[(6-methyl-3,5dicarboethoxy-4-(3-nitrophenyl)1,4- yl)-methyl]-isothiouronium $o$,ó-dibenzoyl-L-tartarate (0.03 g, 30\%) , m.p. $162-163^{\circ} \mathrm{C},[\alpha]_{578}=-29.13^{\circ}(\mathrm{c}=1.3$,MeOH) as yellow crystals.

(-)- S-[(6-methyl)-3,5-dicarboethoxy-4-(3-nitrophenyl)1,4-dihydropyridin-2-yl)-methyl]isothioure (-)-3b. Sodium bicarbonate $(0.01 \mathrm{~g}, 0.12 \mathrm{mmol})$ was added to a suspension of the (-)$4(0.1 \mathrm{~g}, 0.12 \mathrm{mmol})$ in a biphasic $\mathrm{CH}_{2} \mathrm{Cl}_{2} / \mathrm{H}_{2} \mathrm{O}(2: 1,12 \mathrm{ml})$ mixture. The mixture was vigorously stirred for $0.5 \mathrm{~h}$, then the organic phase was separated, washed with water, dried over $\mathrm{Na}_{2} \mathrm{SO}_{4}$ and evaporated. Recrystallization of crude product from $\mathrm{Et}_{2} \mathrm{O}$ furnished (-)-3b (0.03 g, 65\%), mp. $119-120^{\circ} \mathrm{C},[\alpha]_{578}=-10.53^{\circ}\left(\mathrm{c}=1.9, \mathrm{CH}_{2} \mathrm{Cl}_{2}\right)$.

(-)- 2-(Allyl thio)-methyl-3,5-dicarboethoxy-6-methyl-4-(3-nitrophenyl)-1,4-dihydropyridine (-)-3i. An aqueous solution of $\mathrm{NaOH}(32 \%, 0.06 \mathrm{ml})$ was added to a stirred solution of (-)-4 $(0.1 \mathrm{~g}, 0.12 \mathrm{mmol})$ and allyl bromide $(0.03 \mathrm{~g}, 0.29 \mathrm{mmol})$ in ethanol/water $(1: 1,12 \mathrm{ml})$, under an argon atmosphere. After $1.5 \mathrm{~h}$ stirring at room temperature, the mixture was filtered. Recrystallization of the crude product from ethanol furnished compound (-)-3i as yellow needle crystals $(0.02 \mathrm{~g}, 45 \%)$ mp. $118-119^{\circ} \mathrm{C},[\alpha]_{578}=-7.35^{\circ}\left(\mathrm{c}=2, \mathrm{CH}_{2} \mathrm{Cl}_{2}\right)$; $\operatorname{IR}(\mathrm{KBr}) \overline{\mathrm{v}}=3316(\mathrm{~s})$, 3092 (w), 2964-2850 (m), 1675 (s), 1639 (m), 1527 (s), 1350 (s), 1288 (s), 1211 (s), 1101 (s), $802(\mathrm{~s}) \mathrm{cm}^{-1} ;{ }^{1} \mathrm{H} \mathrm{NMR}\left(400 \mathrm{MHz}, \mathrm{CDCl}_{3}\right): \delta=1.22$ (t, $J=7.12 \mathrm{~Hz}, 6 \mathrm{H}, 2 \times \mathrm{CH}_{3}$ ester), $2.41(\mathrm{~s}$, $\left.3 \mathrm{H}, \mathrm{CH}_{3}-6\right), 3.11$ (d, J=7.2 Hz, 2H, S-CH $), 4.01-4.15$ (m, 6H, $2 \times \mathrm{CH}_{2}$ ester, $\mathrm{CH}_{2}-2$ ), 5.00-5.08 $\left(\mathrm{m}, 2 \mathrm{H},=\mathrm{CH}_{2}\right), 5.12(\mathrm{~s}, 1 \mathrm{H}, \mathrm{CH}-4), 5.74-5.82(\mathrm{~m}, 1 \mathrm{H},=\mathrm{CH}), 6.97(\mathrm{~s}, 1 \mathrm{H}, \mathrm{NH}), 7.38(\mathrm{t}, J=7.94$ $\mathrm{Hz}, 1 \mathrm{H}, \mathrm{ArH}) 7.63$ (d, J=7.69 Hz, 1H, ArH), 8.01 (m, 1H, ArH), 8.12 (m, 1H, ArH) ppm. Anal. Calcd for $\mathrm{C}_{22} \mathrm{H}_{26} \mathrm{~N}_{2} \mathrm{O}_{6} \mathrm{~S}: \mathrm{C}, 59.19 ; \mathrm{H}, 5.83 ; \mathrm{N}, 6.27$. Found: C, 58.83; H, 5.89; N, 6.42. 


\section{References}

1. Schleifer, K-J. J. Med. Chem. 1999, 42, 2204.

2. Visentin, S.; Amiel, P.; Frittero, R.; Bpschi, D.; Roussel, C.; Giusta, L.; Carbone, E.; Gasco, A. J. Med. Chem. 1999, 42, 1422.

3. Jiang, J. L.; Li, A.h.; Jang, S.Y.; Chang, L.; Melman, N.; Moro, S.; Ji, X. D.; Lobkowsky, E.; Clardy, J.; Jacobson, K. J. Med. Chem. 1999, 42, 3055.

4. Triggle, D. J.; Lang, D. A. Med. Res. Rev. 1989, 9, 123.

5. Goldman, S.; Stoltefuss, J. Angw. Chem. Int. Ed. 1991, 30, 1559.

6. Hantzsch, A. Justus Liebigs Ann. Chem. 1882, 215, 1.

7. Sircar, I.; Anderson, K. R.; Bonadies, L. Tet. Lett.1988, 29, 6835.

8. Mirzaei, Y. R.; Zenouz, A. M. Iran. J. Chem.\& Chem. Eng.1997, 16(1), 29.

9. Alker, D.; Swanson, A. G. Tet. Lett., 1990, $31(10), 1479$.

10. Young, S. D. Synthesis, 1984, 617.

11. Moshtaghi, Z. A.; Raisossadat O. M.; Mollazadeh, Sh. Synth.Commun. 2005, 35, 2895-2903. 